\title{
Travelers' Malaria
}

Patricia Schlagenhauf-Lawlor

Hamilton, Ontario, Canada: BC Decker Inc, 2001

US \$99.95, 532 pages, hardcover with CD-ROM

Thank you, Dr Schlagenhauf, for writing Travelers' Malaria, a book that many of us did not realize how badly we needed - until you wrote it!

There are lots of physicians, many of them in the field of travel medicine, who have a strong interest in malaria but are not malaria experts and who have only a so-so background in tropical diseases. I am one of them. Twenty-five years ago, when I first became interested in travel and tropical medicine, I could read textbooks and journal articles on malaria and listen to lectures and fairly well understand the gist of the material. But in the last 2 decades, there has been an explosion of knowledge in the field of malaria, as there has been in most other fields of medicine. Increasingly, it requires an extensive background in molecular biology, immunology, biochemistry, and biostatistics to comprehend current malaria material. These are abilities that, sadly, many of us lack. On the other hand, the sections on malaria in most journals and textbooks on travel medicine and infectious diseases are written for individuals with little advanced expertise in the subject, which are therefore not nearly sufficient for the needs of many of us.

Dr Schlagenhauf has assembled about 3 dozen authors, most of them household names in the malaria/ tropical medicine/travel medicine community, and has distilled from their information a well-written and easyto-read, informative, and concise textbook. The book 
stays well within the parameters of the book's name, Travelers' Malaria, but, in addition, and somewhat separately, contains the technical and research material for those needing it. Here is everything that many of us ever wanted to know about malaria for travelers but, in the past, had to go to many sources to find. This book is a real time-saver.

Travelers' Malaria, in a sense, takes a step back and looks at the entire landscape of the disease, from what is known about the beginnings to what we can expect in the future. The beginnings are fraught with controversy. And, as books such as these often do, it bursts bubbles, casting doubt on tales that we accepted as gospel and never questioned. For example, while the early history of malaria is murky, it seems that historians now generally agree that there is little hard evidence to support the stories that the Peruvian Indians were the first to link cinchona bark - the source of quininewith treating malarial fevers. In fact, malaria may not have been present in the New World before Columbus! The disease may have originated in Africa.

As for the present, which is the main thrust of the book, there is a comprehensive chapter on each of the commonly used drugs for chemoprophylaxis and treatment, including the drugs used by the Chinese, and the very effective new drugs, atovaquone/proguanil and tafenoquine. These chapters are ideal for health care providers who counsel travelers visiting malarial areas. The chapter on using mefloquine for chemoprophylaxis has 16 pages plus 98 references. The book makes a case for using doxycycline more often (ie, it argues that this is a very effective and relatively safe antimalarial prophylactic agent and that it is effective in many malarial regions of the world). Each drug chapter has numerous subheadings, making it simple to find the information one seeks. And there are additional chapters that discuss the disease, the drugs, and the treatment in the context of immunocompromised travelers, pregnant women, children, and long-term travelers.

Another chapter discusses the recently developed and widely heralded rapid diagnostic tests, often called dipstick tests. These tests use a drop of blood from a finger stick to diagnose malaria, which is akin to the way in which individuals with diabetes test their blood for glucose levels. The developers of the tests expected that the tests would be widely used by travelers in the field as well as by health care workers not experienced in making blood slides and reading those slides. But experience has shown that test results are often misinterpreted and that occasionally, even high parasitemia levels in the blood are not detected by the tests. Microscopic testing remains mandatory in every patient with the possible diagnosis of malaria.
An important take-away message is that travel health professionals should take more time in educating travelers about how not to get bitten by mosquitoes, and there is much information about how this can be done. Obviously, this is the ideal way to prevent malaria. Although optimum blood levels of specific chemoprophylactic drugs are essential in some malaria areas, the drugs need not be put to the test, so to speak, if the mosquitoes are not allowed to bite. The anopheles mosquito is a fairly predictable foe and can usually be outwitted. In many malarial parts of the world, effective anti-mosquito strategies meticulously followed can reduce the risk of the disease to negligible figures.

Another important message, aimed at primary and emergency physicians, is that no matter where they practice, they should always think malaria. Perhaps 30000 travelers return each year from malarial areas to the developed world infected with the disease. One such case may walk into one's office or emergency room tomorrow. Most physicians in the developed world have never seen a case of malaria (nor have the laboratory technicians who have to read the blood slides). Taking a travel history is essential. And if patients with fever have visited malarial areas, whether they took medication or not, and, sometimes, whether the blood smears for malaria are positive or negative, starting anti-malaria treatment immediately can make the difference between life and death. The 2 chapters titled "Clinical Features of Malaria in Returning Travelers and Migrants" and "Diagnosis of Malaria in Returning Travelers," more than 50 pages, tell the clinician who has never seen a case of the disease how to proceed.

As to the future? Likely, insect control and medications will not prove to be the answer in either eradicating or even controlling the disease. Available chemoprophylactic medications offer good prophylaxis for travelers who can afford the medications and require them for a finite period of time. But only a vaccine will help prevent or reduce the incidence for people living in malaria areas. There are about 300 million new cases of malaria each year worldwide, and about 1 million deaths - these primarily in young children and pregnant women. The person who develops such a vaccine will be given a Nobel Prize in record time. Arguably, only AIDS, and perhaps tuberculosis, are more important infectious diseases to control.

While malaria vaccine development has seen major advancements in the past decade, an effective vaccine is still many years away. Early vaccines targeted the sporozoite stage of the parasite's life cycle, the stage that is injected by the mosquito into humans. These vaccines have been unsuccessful. Current efforts focus on targeting many stages of the life cycle simultaneously and on 
using recombinant proteins or synthetic peptides to maximize the host immune response.

Karl Neumann, MD

Forest Hill, NY, USA 